\title{
La atención primaria de la salud
}

\section{y la especialización médica:}

\section{¿Categorías opuestas o complementarias?}

\author{
Primary care and medical specialization: Complementary \\ or mutually exclusive categories?
}

\author{
Larisa Ivón Carrera, Graciela Teresita Enría, Alberto Enrique D’Ottavio \\ Facultad de Ciencias Médicas y Consejo de Investigaciones, Universidad Nacional de Rosario (UNR), Argentina.
}

Fundamentos y Objetivos: Las conclusiones de Juan César García sobre especialización a fines de los 60 s condujeron a describir aspectos actuales de la especialización; ampliarlos y cotejarlos con aquéllas e indagar las expectativas de los alumnos por ejercer o no en niveles de la Atención Primaria de la Salud - APS-) y su porqué.

Materíal y Métodos: Para ello, fueron combinados métodos cualitativos y cuantitativos en ingresantes, alumnos avanzados y médicos.

Resultados y Discusión: Entre ambas épocas, aumentó la intención por especializarse para el sistema de atención vigente (de 52 a $74 \%$ en los ingresantes y de 66 a $100 \%$ en los alumnos avanzados). En contraste, disminuyó el interés por ser médico/a general de $26 \%$ en los 60s a cerca del $7 \%$ en el 2001. Los ingresantes ignoraban el significado de la APS y sólo $0.5 \%$ de los del ciclo clínico se orientaba hacia ella. La inclinación por especializarse precedía al ingreso, la decisión ocurría en el ciclo clínico (con influencia taxativa de los especialistas) y la elección definitiva acontecía en éste y concluida la carrera, también. Los motivos pueden ser múltiples y cambiantes entre grupos. Las modificaciones contextuales, un sistema de atención anárquico y uno de educación discutible podrían influir. Conclusiones: Valorando a los currículos como coadyuvantes y a las decisiones políticas como determinantes, se infiere que un sistema integral

Correspondencia:

Alberto Enrique D'Ottavio

Matheu 371, 2000 Rosario, Argentina

Fax. 5403414556101

E-mail: aedottavio@hotmail.com

Este trabajo recibió el Premio al Mejor Trabajo en la $V^{a}$ Conferencia Argentina de Educación Médica, La Plata, Argentina. Octubre 2003 de salud y una formación médica compatible podrían proveer salud para todos en un marco de equidad y hacer de la APS y de la especialización categorías complementarias antes que opuestas, como son percibidas actualmente.

Palabras claves: educación médica - especialización - atención primaria - grado - postgrado

Background and Objectives: Juan César García's conclusions on specialization, reported in the late nineteen sixties, encouraged researchers to broaden their area of study and to investigate students' expectations regarding the practice of primary care. Material and Methods: Qualitative and quantitative methods were used to assess the attitudes of new students, advanced undergraduates and postgraduates regarding primary care and specialization.

Results and Discussion: Since the nineteen sixties, the intention to specialize has increased (from 52\% to $\mathbf{7 4 \%}$ in new students and from $66 \%$ to $100 \%$ in advanced undergraduates). The interest in becoming a general physician has fallen from $26 \%$ in the nineteen sixties to approximately $7 \%$ in 2001 . New students are largely unattracted by primary care and only $0.5 \%$ of advanced undergraduates reported interest in this field of medicine. The inclination towards specializing thus precedes entry to medical school, and the actual choice of specialization tends to be made at the clinical stage, where the influence of specialists is particularly noticeable. The definitive choice tends to be made either during this clinical stage or after graduation. The reasons are multiple, and may change from group to group. A chaotic health care system and a debatable educational system may have an influence. Conclusions: Considering curricula as a minor influence 
and political decisions as major influences, we infer that an integral health care system and a compatible medical training could provide health for all in a fair framework and make primary care and medical specialization complementary instead of mutually exclusive, as they are presently perceived.

Key words: medical education - specialization - primary care undergraduate - postgraduate

\section{INTRODUCCIÓN}

La Atención Primaria de la Salud (APS) constituye una estrategia capaz de garantizar la salud para todos y el acceso universal y equitativo al sistema de prestación médica por lo que su implementación requiere de profesionales competentes a fin de desempeñarse con solvencia en cada uno de sus niveles de complejidad ${ }^{1,2}$.

Excediendo el tipo de formato curricular, diferentes facultades de nuestro país han tomado, desde hace ya algunos años, la decisión de formar médicos orientados hacia el primer nivel de complejidad de la $\mathrm{APS}^{3}$.

En un contexto socio-económico-histórico-políticocultural distinto, en el que aún la APS no había sido formulada como la estrategia que hoy representa, el médico argentino Juan César García analizó en los 60s el proceso de especialización en seis Escuelas de Medicina de América Latina (la del Nordeste, Argentina, entre ellas) y 15 de EEUU. Partiendo de la pregunta: “¿Cómo y cuándo se produce en los estudiantes de las sociedades económicamente subdesarrolladas el deseo de especializarse y hacia qué especialidades orientan sus preferencias?", intentó responder tal interrogante a través de encuestas aplicadas a alumnos y a graduados ${ }^{4}$.

Sus conclusiones señalaron que:

- el grado de especialización en los países en vías de desarrollo era elevado; en particular, cuando la prestación de servicios se libraba al juego de la oferta y de la demanda

- la Medicina era la carrera más prestigiosa y existían valoraciones diferenciales entre las especialidades médicas

- los alumnos de Medicina y los médicos de distintos países evidenciaban una significativa similitud en la escala de prestigio adjudicado a las mis- mas especialidades médicas, a la par que minusvaloraban lo psicológico y lo social dentro de la Medicina así como la docencia universitaria y la investigación científica

- la adquisición de tal serie de valores y el deseo por especializarse ocurría previamente al ingreso a la escuela médica, detectándose, en el decurso de la carrera, factores que influían en el prestigio de las especialidades. Así, destacaba que el influjo de la medicina interna ascendía a lo largo de aquélla atribuyéndolo al contacto alumno-especialistas

- el prestigio de las especialidades médicas se sustentaba no sólo en las características intrínsecas de cada una sino en la participación de condiciones locales como la existencia de una destacada escuela de especialistas en el área de influencia

- la clara tendencia hacia la especialización revelada por los alumnos estaba condicionada por circunstancias que escapaban al control de las escuelas médicas

Como colofón, García planteaba la necesidad de profundizar sobre los motivos de la decisión por especializarse pues, agregada a otras razones, conjeturaba una eventual correspondencia entre la distribución de médicos por especialidades en una determinada área y la orientación de las expectativas de los estudiantes a dicho respecto.

Percibidas desde la actualidad, tales conclusiones y la citada hipótesis conducen a nuevos problemas de interés a ser elucidados

En ese marco y en otro contexto socio-económicohistórico-político-cultural (globalización, neoliberalismo y postmodernismo) ${ }^{5}$ se valoró pertinente:

- describir, casi cuarenta años después, aspectos ligados con el proceso de especialización en una escuela de medicina argentina (Facultad de Ciencias Médicas, UNR) para ampliarlas y para cotejarlas con las conclusiones de Juan César García

- indagar las expectativas a futuro de los estudiantes acerca de su ejercicio profesional y los motivos que los conducen a elegir ejercer como médicos orientados hacia el primer nivel de complejidad de la APS; como especialistas en otro de sus niveles o bien como especialistas dentro del sistema de atención médica vigente

- comparar los resultados con el registro de especialidades y especialistas del Colegio de Médicos de la $2^{\text {a }}$ Circunscripción (siete departamentos de la zona sur de la Provincia de Santa Fe, Argentina) como aporte a la hipótesis formulada por García 


\section{MATERIAL Y MÉTODOS}

Para alcanzar los objetivos se empleó una combinación de métodos

\section{Método cualitativo ${ }^{6}$}

Con la intención de describir aspectos del proceso de toma de decisiones e interpretar las valoraciones intrínsecas transformadas en prácticas, fueron implementadas dos técnicas:

Historias de vida en virtud de las potencialidades de las historias de vida para recoger la narración de lo vivido y vivenciado, fueron entrevistados diez médicos, con notoria motivación, implicación y compromiso, cuyas carreras académicas y profesionales se mostraran diferentes

Grupos focales: integrados por alumnos de Medicina y médicos que evidenciaron interés por participar de las reuniones. Cada uno de los grupos fue coordinado por un investigador, quien facilitó la discusión y tomó en cuenta, a modo de ejes: las valoraciones sobre la profesión médica, la elección de las especialidades, los momentos en que los participantes debieron tomar decisiones de relevancia y las razones que los condujeron a dichas elecciones

Las narraciones, recogidas en cintas magnetofónicas, fueron decodificadas textualmente constituyéndose en los indicadores a partir de los que resultó posible interpretar los referentes que llevaron a la toma de decisiones y a sus consecuencias.

Ambas instancias permitieron construir categorías contrastables del tema indagado que actualizaron el cuestionario original de García.

\section{Método cuantitativo}

Se realizó una encuesta poblacional mediante un cuestionario, válido y fiable, superponible y actualizado respecto del oportunamente empleado por García e integrado por preguntas cerradas y abiertas. Estas últimas, susceptibles de abrir las posibilidades descriptivas de las variables, fueron codificadas y tratadas como cerradas en el momento del procesamiento.

Dicho cuestionario examinó la valoración del ser médico y sus cualidades representativas, destacándose facetas vinculadas con la especialización, compatibles con aquéllas de la década del 60 . Cabe destacar, entre ellas, el ordenamiento de especialidades según su prestigio dentro de la profesión médica y a opinión del encuestado, para cuyo procesamiento se usó el mismo score entonces empleado.

La encuesta fue aplicada de manera aleatoria, durante el 2001 (último año con currículo tradicional en nuestra Facultad y similar al vigente durante el estudio de los 60), a 512 estudiantes de primer año de la carrera ( $25 \%$ del total de los inscriptos por primera vez) [270 mujeres y 242 varones], a 240 de su cuarto y quinto año ( $23 \%$ del total) [123 mujeres y 117 varones] y a 45 médicos a tres años de su graduación (15\% del total) [27 mujeres y 18 varones]. Se completó con ello un total de 797 encuestas, cuya aplicación siguió estrictas normas éticas e hizo inviables las mutuas interacciones entre los participantes voluntarios.

Además, se obtuvo información referida a las especialidades y a los especialistas de la base de datos del Colegio de Médicos ( $2^{\mathrm{a}}$ Circunscripción, Santa $\mathrm{Fe}$ ) con el objeto de ponderar su influencia en los aspectos indagados.

A fin de validar los resultados, se procedió al análisis de los mismos y al cotejo de lo hallado por nosotros con lo obtenido por García así como con los datos provistos por dicho Colegio.

El estudio estadístico requirió del cálculo de promedios \pm desvíos estándares y del uso del test de $\mathrm{x}^{2}$.

Finalmente, la combinación de métodos procuró aumentar la confianza de los resultados y enriquecer las explicaciones sobre el tema en cuestión en favor de un enfoque más holístico aunque intentando que ni uno ni otro se transformaran en meras cortinas en favor de alguno de ellos.

\section{RESULTADOS}

El $74 \%$ de los 512 estudiantes encuestados de primer año (edad $=18 \pm 1)$, ninguno de los cuales manifestó conocer el significado de la APS ni se orientó hacia el sanitarismo, pensaba en especializarse mientras que de los 240 alumnos/as de cuarto y quinto año (edad $=24 \pm 2)$, de los que sólo el $0.5 \%$ se orientó hacia el primer nivel de complejidad de la APS y ninguno hacia el sanitarismo, el $100 \%$ se había decidido ya por especializarse.

La Tabla 1 recopila en valores absolutos y porcentajes, según sexo y ciclo cursado de la carrera, las preferencias de los alumnos por distintas especialidades médicas.

De la Tabla 1 se destacan fundamentalmente:

1. las preferencias por Cirugía respecto de la Medicina Interna, en el ciclo básico, se invierten en el ciclo clínico y resultan ambas altamente significativas al compararse sus totales del básico versus sus totales del clínico ( $p<0.001$ en los dos casos). En este último, además, se visualizan diferencias significativas entre sexos tanto en Cirugía cuanto en Medicina Interna $(p<0.001$ en una y otra) 
Tabla 1. Preferencias de los alumnos, según sexo y cido cursado de la carrera, por diferentes especialidades médicas (en valores absolutos y porcentajes)

\begin{tabular}{|c|c|c|c|c|}
\hline \multirow{3}{*}{$\begin{array}{l}\text { Año/s de la carrera médica } \\
\text { Cirugía }\end{array}$} & \multicolumn{2}{|c|}{$\begin{array}{l}\text { ler.año } \\
\text { (ciclo básico médico) }\end{array}$} & \multicolumn{2}{|c|}{$\begin{array}{l}\text { Años } 4^{\circ} \text { y } 5^{\circ} \\
\text { (ciclo clínico médico) }\end{array}$} \\
\hline & \multicolumn{2}{|r|}{ M } & $\mathrm{V}$ & $\mathrm{M}$ \\
\hline & $\begin{array}{l}97 \\
(40 \%)\end{array}$ & $\begin{array}{l}103 \\
(38 \%)\end{array}$ & $\begin{array}{l}27 \\
(23 \%)\end{array}$ & $\begin{array}{l}14 \\
(11 \%)\end{array}$ \\
\hline Medicina Interna & $\begin{array}{l}56 \\
(23 \%)\end{array}$ & $\begin{array}{l}68 \\
(25 \%)\end{array}$ & $\begin{array}{l}43 \\
(37 \%)\end{array}$ & $\begin{array}{l}62 \\
(50 \%)\end{array}$ \\
\hline Medicina General & $\begin{array}{l}19 \\
(8 \%)\end{array}$ & $\begin{array}{l}22 \\
(8 \%)\end{array}$ & $\begin{array}{l}6 \\
(5 \%)\end{array}$ & $\begin{array}{l}6 \\
(5 \%)\end{array}$ \\
\hline Especialidades Clínicas & $\begin{array}{l}20 \\
(8 \%)\end{array}$ & $\begin{array}{l}43 \\
(16 \%)\end{array}$ & $\begin{array}{l}16 \\
(14 \%)\end{array}$ & $\begin{array}{l}21 \\
(17 \%)\end{array}$ \\
\hline Especialidades Quirúrgicas & $\begin{array}{l}36 \\
(15 \%)\end{array}$ & $\begin{array}{l}19 \\
(7 \%)\end{array}$ & $\begin{array}{l}21 \\
(18 \%)\end{array}$ & $\begin{array}{l}16 \\
(13 \%)\end{array}$ \\
\hline Investigación Científica & $\begin{array}{l}7 \\
(3 \%)\end{array}$ & $\begin{array}{l}8 \\
(3 \%)\end{array}$ & $\begin{array}{l}1 \\
(1 \%)\end{array}$ & (1 \%) \\
\hline Docencia Universitaria & $\begin{array}{l}5 \\
(2 \%)\end{array}$ & $\begin{array}{l}5 \\
(2 \%)\end{array}$ & $\begin{array}{l}1 \\
(1 \%)\end{array}$ & $\begin{array}{l}1 \\
(1 \%)\end{array}$ \\
\hline Salud Mental & $\begin{array}{l}1 \\
(0.5 \%)\end{array}$ & $\begin{array}{l}1 \\
(0.5 \%)\end{array}$ & $\begin{array}{l}1 \\
(1 \%)\end{array}$ & $1 \%$ \\
\hline Medicina Social & $\begin{array}{l}1 \\
(0.5 \%)\end{array}$ & $\begin{array}{l}1 \\
(0.5 \%)\end{array}$ & $\begin{array}{l}1 \\
(1 \%)\end{array}$ & $\begin{array}{l}1 \\
(1 \%)\end{array}$ \\
\hline Totales & 242 & 270 & 117 & 123 \\
\hline
\end{tabular}

2. un descenso de interés por la Medicina General en el ciclo clínico, a partir de una ya baja inclinación por ella en 1er. año

3. una repartida afinidad por las especialidades quirúrgicas y médicas en ambos ciclos, con diferencias intersexuales significativas sólo en el básico ( $\mathrm{p}$ $<0.01$ en ambos casos)

4. una escasa orientación inicial por la investigación científica y la docencia universitaria en declinación diacrónica

5. una estable y bajísima opción por la salud mental y la medicina social en uno y otro ciclo

Los médicos (edad $=30 \pm 2$ ) reprodujeron los resultados obtenidos en los alumnos del ciclo clínico.
En relación con los distintos momentos del proceso de decisión, si bien el $100 \%$ de los encuestados valoró la posibilidad de especializarse previamente al ingreso a la carrera; la decisión definitiva fue adoptada, en mujeres y varones, durante el ciclo clínico y la elección de la especialidad, también en ambos sexos, hecha por el $75 \%$ en el ciclo clínico y por el 25 $\%$ restante durante el medicato (1). Estos resultados fueron corroborados por los dichos de los médicos.

A su vez, los motivos de elección de las especialidades no evidenciaron diferencias estadísticas significativas intersexuales aunque mostraron categorías y porcentajes de adhesión diferentes según el ciclo cursado de la carrera médica (Tabla 2)

Tabla 2. Categorias y porcentajes de adhesión referidos a los motivos de elección de las especialidades durante la carrera médica

\begin{tabular}{|c|c|c|}
\hline Alumnos de ler. año (ciclo básico médico) & \multicolumn{2}{|c|}{ Alumnos de $4^{\circ}$ y $5^{\circ}$ año (ciclo clínico médico) } \\
\hline Necesidad de $4^{\circ}$ nivel instruccional (42\%) & Seguridad económico-laboral & $(39 \%)$ \\
\hline Modelos cercanos y mass media $\quad(31 \%)$ & Influencia de docentes-especialistas & $(32 \%)$ \\
\hline Relevancia asignada a la Anatomía (19\%) & Especialidades c/ aporte tecnológico & $(15 \%)$ \\
\hline \multirow[t]{3}{*}{ Otros } & Especialidades de futuro & $(6 \%)$ \\
\hline & Especialidades de moda. & $(5 \%)$ \\
\hline & Afamados especialistas locales & $(3 \%)$ \\
\hline
\end{tabular}

1. Forma de práctica optativa ofrecida por la Facultad de Ciencias Médicas, UNR a los graduados y realizada en hospitales provinciales y municipales 
Los médicos repitieron prácticamente los motivos citados por los alumnos del ciclo clínico. Un grupo de encuestados expresó que cambió su elección original forzado por la imposibilidad de acceder a la especialidad elegida en primera instancia (2).

Con referencia al puntaje promedio otorgado por los encuestados a las especialidades de acuerdo con el prestigio social asignado por la profesión médica, se obtuvo el orden de prelación que se lista, siguiendo el score propuesto por García que otorga mayor prestigio social a menor puntaje (3):

a. entre 0 y 2: Cirugía y Medicina Interna

b. entre 4 y 6: Pediatría, Neurología, Ginecología, Obstetricia y Oftalmología

c. entre 8 y 10: Medicina Preventiva y Social Otorrinolaringología - Dermatología - Salud Pública, Docencia e Investigación

No se produjeron diferencias significativas en las valoraciones apuntadas entre grupos ni entre sexos $(\mathrm{p}>0.05)$.

Por otra parte, de los casi 8000 médicos inscriptos en el Colegio de Médicos (2a Circunscripción) (61\% varones) hay 5000 especialistas (64\% varones) y de las 68 especialidades, las más pobladas son, en estricto orden: Pediatría, Medicina Interna, Cardiología, Cirugía General, Psiquiatría, Traumatología y Ortopedia, Ginecología, Anestesiología y Obstetricia. Cabe resaltar que, reconocida como especialidad por el Colegio de Médicos, la medicina general y familiar recluta a la fecha (4) sólo 25 especialistas (14 mujeres y 11 varones).

$\mathrm{Al}$ cotejar esta realidad ordinal con el listado valorativo y con las especialidades pensadas y elegidas por los alumnos, aparece una cierta correspondencia que no puede pasar inadvertida.

Finalmente, aunque casi el $100 \%$ de los encuestados (varones y mujeres) pensó en otra especialidad alternativa, la mayoría se expresó en favor de la elegida o de la ejercida al momento de la encuesta. Más aún, una importante proporción de médicos (5), de uno y otro sexo, reveló como muy acertada la elección de su especialidad como medio y modo de vida, considerando que ésta le proveía la mayor satisfacción.

\section{DISCUSIÓN}

\section{Sobre la especialización}

En el presente trabajo, la especialización se mostró como la opción de formación y de ejercicio profesional más valorada desde el comienzo de la carrera. Así, en el 2001, los jóvenes que se decidieron por Medicina expresaban su intención por especializarse desde su ingreso a la carrera (74 \%) hasta fines de ella (100\%), superando con holgura los porcentajes de la década del $60(52 \%$ y $66 \%$, respectivamente). Después de veinte años de lanzada la propuesta de APS, apenas un $8 \%$ de los alumnos de 1er. año y un $5 \%$ de los alumnos de $4^{\circ}$ y $5^{\circ}$ año (6) se inclinaban por la medicina general, a diferencia de lo ocurrido en los sesenta cuando tal porcentaje se elevaba al $26 \%$. Entre las épocas cotejadas, las preferencias por la medicina vinculada con lo social y con la salud mental (7) se mantuvieron escasas mientras el sanitarismo, en cualquiera de sus propuestas (8), fue ignorado por completo. A su vez, la investigación y la docencia universitarias recibieron exiguas y descendentes adhesiones (investigación: $8 \%$ en los $60 \mathrm{~s}, 3 \%$ en alumnos de $1 \mathrm{er}$ año (2001) y $1 \%$ en alumnos de $4^{\circ}$ y $5^{\circ}$ año (2001) y docencia: $2 \%, 2 \%$ y $1 \%$, respectivamente). En esto último, podrían estar influyendo los bajos salarios, los riesgos que implica apostar todo a una dedicación exclusiva, un sistema de educación médica que hace escaso hincapié en ellas así como la ausencia de políticas definidas en favor de una y de otra.

La valoración por la cura y, en particular, por la extirpación radical del mal registró un aumento ya que, en el 2001, prácticamente el $40 \%$ de los ingresantes a Medicina pensaba especializarse en Cirugía respecto del $11 \%$ de los 60 s. Por su parte, y como en aquella década, se repitió la elevación del prestigio de la Medicina Interna a lo largo de la carrera médica. Sin embargo, en la actualidad y a diferencia de entonces cuando nadie refirió pensar en ella como especialidad, ese aumento de prestigio estuvo acompañado por un incremento significativo de quienes deseaban especializarse en la misma tanto en los estudiantes de 1er. año cuanto en los del ciclo clínico. Corresponde señalar aquí que, si

2. Tres de veintiuno $3 / 21$

3. JC García, página 213, 1972

4. Junio del 2003

5. Diecisiete de veintiuno $17 / 21$

6. No se citan en este apartado los resultados obtenidos en médicos por ser prácticamente superponibles con los de los alumnos del ciclo clínico

7. Llamativamente la Psiquiatría ocupa el $5^{\circ}$ lugar en el número de especialistas del Colegio de Médicos de la $2^{\mathrm{a}}$ Circunscripción (Santa Fe)

8. Salud pública, epidemiología, medicina preventiva, medicina social. Se lo analiza en Especialización y APS. 
bien los alumnos actuales se manifiestan crecientemente atraídos por la Medicina Interna, ésta les genera, a la par, definidas prevenciones por su progresiva magnitud abarcadora. En relación con la diferencia a favor de las mujeres detectada a este respecto en el ciclo clínico, en la misma podría estar actuando la progresiva feminización de la matrícula médica, que - aunque escapa a los objetivos de este trabajo - se está estudiando en profundidad.

En parecido sentido, cabría preguntarse si el ya citado descenso en la inclinación femenina por la Cirugía obedece únicamente a los motivos de elección esgrimidos o si, además, responde a que el acceso a la misma suele hallarse obstaculizado por diferentes motivos. En consonancia con ello, resulta llamativa la creciente inclinación de las mujeres por las especialidades quirúrgicas como ginecología y oftalmología, entre otras.

Los determinantes de este particular aumento por la especialización pueden hallarse en el contexto socio-económico-histórico-político-cultural (globalización, neoliberalismo, postmodernismo) con su instalado paradigma de la economía de la salud que se traduce en la estructura anárquica, fragmentada y escotomizada (9) de la atención de la enfermedad sin posibilidades de ser integrada en un sistema de complejidad creciente que contemple las diferentes problemáticas poblacionales (10). Esto fortalece las representaciones sociales que, acerca del ejercicio profesional, tienen los estudiantes, quienes - al "leer mejor la realidad que los textos, las instrucciones en los transparentes o las consignas de las evaluaciones" al decir de Jaim Etcheverry ${ }^{7}$ - buscan nichos prometedores de una mejor inserción laboral y de una mayor seguridad económica, muchas veces más supuestas que reales. En sintonía con ello, lo dicho constituye un impedimento a los denodados esfuerzos del sistema educativo por revertir tal situación.

Si bien, de acuerdo con García, la adquisición de la escala de prestigio adjudicado a las especialidades médicas y el deseo por especializarse ocurriría previamente al ingreso a la escuela médica y recibiría posibles influencias de especialistas dentro de ella, en este trabajo corroboramos que el momento del pensamiento inicial se ubicaría antes del ingreso a la misma y, además, ponemos de relieve que la decisión por una especialidad se produciría en los años finales del ciclo clínico, con influencia taxativa de docentes-especialistas, entre otros, y que la definición final se haría en dicho ciclo y aún, ya concluida la carrera, en consonancia con un largo período deliberativo en el que fueron confrontadas ventajas y desventajas ${ }^{8,9,10,11}$.

Los motivos de elección aquí caracterizados muestran un atrayente grado de coincidencia con las razones esgrimidas entre los 60s y la actualidad en la decisión por Medicina (teóricas en las 60s y tecnológicas en el 2001), proceso que estudiáramos anteriormente. Además, la eventual influencia de escuelas afamadas de especialistas ya había sido arriesgada por García. También vinculado con este ítem, y más allá de su escaso número, debe tenerse en cuenta, recordando a Almafuerte, que "no todos hieren la cuerda que quieren" ${ }^{12}$ desde que hubo quienes debieron cambiar su elección original forzados por la imposibilidad de acceder a la especialidad elegida en primera instancia.

A su vez, ciertas correspondencias que pudimos detectar entre las especialidades elegidas, la escala valorativa asignada por alumnos y graduados a las distintas especialidades y el número de especialistas en la zona de directa influencia de nuestra Facultad de Ciencias Médicas, aportan datos favorables a la vinculación conjeturada por García en los 60s.

Los resultados comunicados confirman, a la fecha, la divergencia, señalada ya por el mismo autor, entre lo que planifican las escuelas médicas y lo que deciden a la postre sus alumnos pues - según él- la especialización se hallaría influenciada por factores sobre los que las escuelas carecen de control.

Por eso, los casi cuarenta años transcurridos desde su estudio original, parecen darle la razón cuando decía “... Las escuelas de medicina establecen metas que no pueden alcanzar, basándose en la creencia de que la educación puede cambiar procesos que dependen, en realidad, de factores económicos y/o de decisiones políticas". Por su parte, Paganini asevera en la actualidad que el sector Salud debe revitalizarse, que las ciencias de la Salud Colectiva han perdido protagonismo y que la economía les ha impuesto sus reglas de juego y evoca, en tal sentido, las ideas predominantes en este tema desde los años 60 hasta el presente, esto es, desde la Extensión de Cobertura hasta la Economía de la Salud pasando por ese mensaje de equidad al que le faltó instrumentación- Salud para Todos - y la Promoción de la Salud) ${ }^{13,14}$.

9. Especializadas en porciones del organismo humano.

10. Expresadas en sus diferentes patrones y perfiles patológicos. 
Finalmente, la Medicina sigue siendo considerada a la fecha una carrera prestigiosa, a pesar de su proletarización, y con valoraciones diferenciales de sus especialidades tradicionales, a las que se han sumado otras nuevas, de resultas del avance científico-tecnológico, y sub-especialidades que otorgan valor agregado y son objeto de la búsqueda de nuevas formas en el ejercicio profesional, en progresiva deshumanización.

\section{Sobre la APS y la especialización}

Un recorrido por las conclusiones de Juan César García y por los resultados aquí reportados, con casi cuatro décadas de diferencia, nos brinda prima facie una visión aflictiva, pues si bien la APS y la especialización médica no son necesariamente categorías antitéticas, parecieran serlo a la luz de: (a) los porcentajes nulos por el sanitarismo, ayer y hoy; (b) la minusvaloración que los alumnos de Medicina y los médicos suelen realizar de lo psicológico, lo epidemiológico y lo social dentro de la Medicina, siendo claves como son para abordar problemas poblacionales; (c) la sensible disminución, más de veinte años después de lanzada la APS, del interés por la medicina general (que, reiteramos, se redujo del $26 \%$, en la década del 60 , al 8 $\%$ en alumnos de $1^{\circ}$ y al $5 \%$ en los del ciclo clínico, durante el 2001); (d) la bajísima cantidad de/ médicos generales en la $2^{\text {a }}$ Circunscripción médica santafesina, donde son considerados como especialistas; (e) la circunstancia, compatible con el ítem anterior, de constituir la inscripción a esta especialidad una opción alternativa, y (f) la ignorancia de los ingresantes sobre el significado de la APS y la reducidísima cantidad de alumnos que se orientaba hacia el primer nivel de complejidad de la APS en el ciclo clínico.

Evidentemente los estudiantes no perciben todavía con claridad su ejercicio profesional como médicos orientados hacia el primer nivel de complejidad de la APS. Esta decisión no estaría aún instalada en ellos probablemente porque, excediendo su estímulo en magnitud diferencial desde los currículos, sea precisa una decisión política de Estado que viabilice un sistema integral de atención médica capaz de ofrecerles una buena inserción laboral, remuneraciones dignas y posibilidades ciertas de mejoramiento profesional.

En realidad, los currículos podrían oficiar de coadyuvantes ya que, en congruencia con lo aseverado por García, la educación médica no puede cambiar ad integrum procesos que dependen prioritariamente de decisiones políticas.

En síntesis, aunque a García le sorprendiera ingratamente que apenas un $24 \%$ de estudiantes se inclinara por la medicina general, este estudio, hecho en un contexto socio-económico-históricopolítico-cultural distinto, revela una disminución sustantiva al respecto; más aún, si se reitera que el $100 \%$ de los alumnos del ciclo clínico está decidido por una especialidad, que de ellos muy pocos desean hacerlo en medicina general y que los médicos generales, según los datos provistos por el Colegio Médico de la $2^{\mathrm{a}}$ Circunscripción que los reconoce como especialistas, son escasísimos a la fecha en la parte sur de la provincia argentina de Santa Fe.

$\mathrm{Al}$ respecto y como sustento de tal disminución, no puede soslayarse que este trabajo fue realizado después de Alma Ata (Kazajstán, ex -URSS, 1978) con su innovadora propuesta de atención primaria de la salud, posteriormente acotada; después del lema Salud para Todos en el año 2000; en el contexto globalizado, neoliberal y postmoderno que sucediera a la disolución de la URSS (1989) y en el curso de un currículo orientado hacia la consecución de un médico general.

Por último, el cotejo con la bibliografía internacional mostró ciertas coincidencias que indican la universalidad del problema en cuestión así como diferencias propias de factores locales que no pueden ser descartados ${ }^{15,16,17,18,19}$.

Considerando la totalidad de lo analizado, las necesidades actuales para garantizar salud para todos y acceso universal y equitativo al sistema de prestación médica así como el empeño puesto por muchas escuelas médicas para formar médicos/as generales, aptos/as para APS, nuevas indagaciones permitirán ponderar si los currículos que pusieron mayor énfasis en la APS lograron modificaciones cuantitativas y cualitativas sustanciales respecto del modo elegido de ejercicio profesional, a través de la formación de médicos convencidos de la necesidad de concretar un modelo de APS que garantice la promoción de la salud, la prevención de las enfermedades, la curación y rehabilitación con real participación de los diferentes grupos sociales.

Ello, además de abogar en favor del papel coadyuvante concedido a la educación médica frente al rol determinante que, en coincidencia con García, se asigna a las decisiones políticas gubernamentales, evidenciaría que una apropiada estrategia, como la implementación de un sistema integral de salud con una formación médica compatible anexa, puede viabilizar una cobertura sanitaria universal en Argentina y hacer de la APS y de la especialización categorías complementarias antes que opuestas, como son percibidas en la actualidad. 


\section{AGRADECIMIENTOS}

Los autores testimonian su profundo agradecimiento al Prof. Juan Carlos Miguel y al Colegio de Médicos de la $2^{\text {a }}$ Circunscripción de la Provincia de Santa Fe por los valiosos aportes realizados.

\section{BIBLIOGRAFÍA}

1. Enria, G. Staffolani C: Participación Comunitaria en Salud ¿Utopía o Compromiso Político? Anuario Fundación Dr. J. R. Villavicencio: 28-34, 2001

2. Enria, G. Staffolani C: Atención Primaria de la Salud: Obstáculos para su concreción. III Congreso Internacional de Medicina General XI Congreso del equipo de Salud, Santa Fe (Argentina), 2001

3. D’Ottavio AE: Ser Médico. Reflexiones sobre la formación y la práctica médicas. Homo Sapiens ediciones. Rosario (Argentina), 2002

4. García JC. La Educación Médica en América Latina. Oficina Panamericana de la Salud. Publicación Científica $\mathrm{N}^{\circ} 255$, Washington DC (USA), 1972

5. Díaz E (editora): La Posciencia (El conocimiento científico en las postrimerías de la modernidad). Editorial Biblos, Buenos Aires (Argentina), 2000

6. González Rey F: La investigación cualitativa en Psicología: rumbos y desafíos. Editorial EDUC, San Pablo (Brasil), 1999

7. Jaim Etcheverry G: La Tragedia Educativa. Fondo de Cultura Económica. Buenos Aires (Argentina), 1999

8. Carrera LI et al: Study of the decision making process for the medical career. Biocell: 27: 2: 227, 2003
9. D'Ottavio AE et al: Why Argentinean students studied Medicine then and now. Acad Med 72: 833-834, 1997

10. Miguel JC \& D'Ottavio, AE: Postgraduate specialization of Argentinean medical students. Acad Med 73: 1029-1030, 1998

11. AFACIMERA Curso a Distancia: Desafíos y Tendencias. Módulo No 3, 37-40, 1997/98

12. Almafuerte: Mater Dolorosa. En Obras Comple-tas, Antonio Zamora ediciones. Buenos Aires (Argentina), 333-334, 1975

13. Ferrara F, Acebal E \& Paganini JL: Medicina de la Comunidad (Medicina Preventiva, Medicina Social y Medicina Administrativa). Editorial Interamericana, Buenos Aires (Argentina), 1972

14. Paganini JL: La atención primaria como estrategia de cambio curricular en la formación médica. html pdf, 2002

15. Kassebaum DG et al: Factors influencing the specialty choices of 1992 medical school graduates Acad Med 69: 163-170, 1994

16. International Medical Workforce Conference: The overlapping roles of primary care physicians, general specialists and sub-specialists. Oxford, UK, 2003

17. Kucharz EJ: Internal medicine: yesterday, today, and tomorrow III. Specialists versus generalists or hospitalists European Journal of Internal Medicine 14:3:205-208, 2003; 14:4: 272-274, 2003;14:5: 344-346, 2003

18. Liev TA et al.: Specialty choices at one medical school: recent trends and analysis of predictive factors. Acad Med 64: 622629,1989

19. Moore G et al: Primary care medicine in crisis: toward reconstruction and renewal. Ann Intern Med 138: 244-247, 2003 